\title{
Prediction of thickness limits of ideal polar ultrathin films
}

\author{
Zhicheng Zhong," G. Koster, and Paul J. Kelly \\ Faculty of Science and Technology and MESA ${ }^{+}$Institute for Nanotechnology, University of Twente, P.O. Box 217, \\ 7500 AE Enschede, The Netherlands
}

(Received 6 July 2011; revised manuscript received 5 March 2012; published 26 March 2012)

\begin{abstract}
Competition between electronic and atomic reconstruction is a constantly recurring theme in transition-metal oxides. We use density functional theory calculations to study this competition for a model system consisting of a thin film of the polar, infinite-layer structure $A \mathrm{CuO}_{2}(A=\mathrm{Ca}, \mathrm{Sr}, \mathrm{Ba})$ grown on a nonpolar, perovskite $\mathrm{SrTiO}_{3}$ substrate. A transition from the bulk planar structure to a chain-type thin film accompanied by substantial changes to the electronic structure is predicted for a $\mathrm{SrCuO}_{2}$ film fewer than five unit cells thick. An analytical model explains why atomic reconstruction becomes more favorable than electronic reconstruction as the film becomes thinner, and suggests that similar considerations should be valid for other polar films.
\end{abstract}

DOI: 10.1103/PhysRevB.85.121411

PACS number(s): 68.55.-a, 68.35.Ct, 81.15.Aa, 73.20.-r

Introduction. The lowering of symmetry at surfaces and interfaces frequently results in new or enhanced physical properties. A central goal of transition-metal oxide thin-film engineering is to exploit this by making interface effects dominate bulk properties in a controlled fashion. ${ }^{1,2}$ When a polar thin film is grown on a nonpolar substrate, achieving this control can be very difficult because an instability arises that can drive electronic and atomic reconstruction. In the case of $\mathrm{LaAlO}_{3}$ (LAO) thin films grown on $\mathrm{SrTiO}_{3}$ (STO) substrates, ${ }^{2-5}$ the alternate stacking of positively $\left(\mathrm{LaO}^{+}\right)$and negatively $\left(\mathrm{AlO}_{2}{ }^{-}\right)$charged layers should result in huge internal electric fields. If nothing else were to happen, the increasing electrostatic energy would give rise to a "polar electrostatic instability". The displacement in response to this instability ${ }^{5,6}$ of electrons (charge transfer or leakage) and ions (atomic relaxation and reconstruction) can generate compensating electric fields. Whereas charge transfer and atomic relaxation result in atomically sharp interfaces and ideal thin films with essentially intrinsic properties, ${ }^{7-13}$ atomic reconstruction can result in the formation of structures with entirely different intrinsic properties, or in changes to the local stoichiometry due to, e.g., oxygen vacancy formation ${ }^{14}$ or ionic intermixing. ${ }^{15,16}$ The competition between electronic charge transfer and atomic relaxation and reconstruction in oxide thin films is the source of much puzzling behavior. . $^{5-16}$

To unravel the details of this competition is a challenge for experiments because of the limited resolution of interfacesensitive measurements and the low visibility of oxygen atoms in most techniques; ${ }^{5}$ it is also a challenge for theoretical studies to describe atomic reconstruction that results from subtly different experimental conditions ${ }^{13,17}$ when the role of stoichiometry is unclear. In this Rapid Communication, we use first-principles total energy calculations to study the growth of thin films of the polar infinite-layer copper oxide $A \mathrm{CuO}_{2}$ ( $A=\mathrm{Ca}, \mathrm{Sr}, \mathrm{Ba})$ on a nonpolar perovskite STO substrate and predict a stoichiometric atomic reconstruction as a function of the film thickness. As the parent compound of cuprate high-temperature superconductors (HTSs), $A \mathrm{CuO}_{2}(A C \mathrm{C})$ has been intensively studied. ${ }^{18-21}$ The recent discovery of very high mobilities in heterostructures containing thin films of $\mathrm{SrCuO}_{2}(\mathrm{SCO})^{22}$ and a theoretical proposal for electron-hole liquids $^{23}$ has led us to reexamine its structural and electronic properties. Our finding of chain-type formation in thin films as a result of an electrostatic instability and atomic reconstruction provides important insight into the growth of thin films of cuprate HTSs. We note that the chain-type formation is different from a $\mathrm{GdFeO}_{3}$ type $^{9}$ or a polar ${ }^{11}$ distortion in LAO|STO, ferroelectric or antiferrodistortive distortions in $\mathrm{PbTiO}_{3} \mid \mathrm{SrTiO}_{3},{ }^{24}$ and in bulk $\mathrm{Ca}_{3} \mathrm{Mn}_{2} \mathrm{O}_{7} \cdot{ }^{25}$

Method. We study ACO thin films in three forms: grown on an STO substrate; in ACO|STO multilayers; and freestanding. The thickness of $A C O$ is varied from one to six unit cells while keeping the STO thickness fixed at five unit cells. The in-plane lattice constants of all thin films are fixed at the equilibrium value of the bulk STO substrate calculated to be $a_{\text {STO }}=3.87 \AA$ and all atoms are allowed to relax fully. The local density approximation (LDA) calculations were carried out with the projector augmented-wave method ${ }^{26}$ as implemented in the Vienna $A b$ initio Simulation Package (VASP). ${ }^{27}$ Using the generalized gradient approximation (GGA) does not change our main conclusions. To better describe localized $\mathrm{Cu} d$ electrons, we use the rotationally invariant LDA $+U$ method $^{28}$ with $U-J=6.5 \mathrm{eV}^{29}$

Bulk materials. Bulk STO is a well-studied band insulator and a popular substrate for growing thin oxide films. It has a typical perovskite structure in which $\mathrm{Sr}$ and $\mathrm{TiO}_{6}$ form a $\mathrm{CsCl}$ lattice, and the $\mathrm{TiO}_{6}$ unit consists of an oxygen octahedron with $\mathrm{Ti}$ at its center and the oxygen atoms at the centers of the Sr cube faces. Assigning the formal ionic charges $\mathrm{Sr}^{2+}, \mathrm{Ti}^{4+}$, and $\mathrm{O}^{2-}$, it can also be described as an alternate stacking of uncharged $\mathrm{SrO}^{0}$ and $\mathrm{TiO}_{2}{ }^{0}$ layers. ${ }^{2}$

The copper oxide $A \mathrm{CuO}_{2}$ with the infinite-layer structure ${ }^{18}$ depicted in Fig. 1 can be regarded as a defect perovskite with ordered oxygen vacancies. It consists of positively charged $A^{2+}$ and negatively charged $\mathrm{CuO}_{2}{ }^{2-}$ layers. Because all of the oxygen sites in the $A \mathrm{O}$ plane are vacant, there are $\mathrm{Cu}-\mathrm{O}$ bonds in the $x y$ plane but none in the $z$ direction. The missing atoms and bonds lead to a reduction of the lattice constant in the $z$ direction, reducing the symmetry from cubic in STO to tetragonal in ACO. The lattice constant of ACO increases as the ion radius increases in the series $A=\mathrm{Ca}, \mathrm{Sr}, \mathrm{Ba}$. The calculated values given in Table I agree well with experiment and SCO is seen to have the smallest lattice mismatch with STO. Though a complex orthorhombic structure for SCO can 


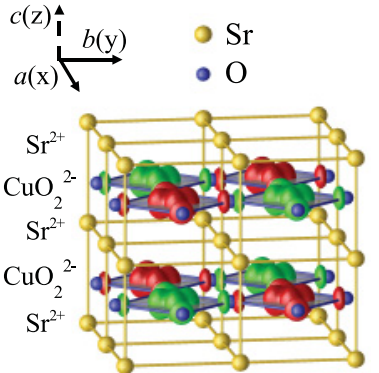

Bulk $\mathrm{SrCuO}_{2}$

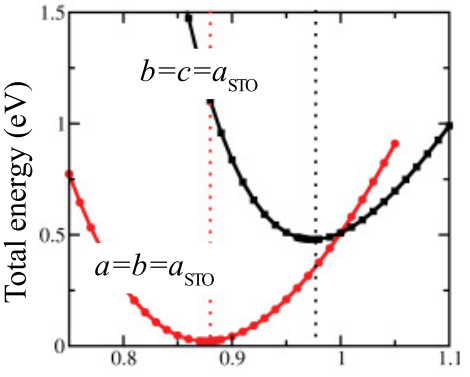

Lattice constant (in units of $a_{\mathrm{STO}}$ )
FIG. 1. (Color online) Left-hand panel: Bulk $\mathrm{SrCuO}_{2}$ with infinite-layer structure. Dark gray (red) and gray (green) represent surfaces of constant magnetization density but of opposite sign. Right-hand panel: The dark gray (red) curve illustrates the strain energy of bulk $\mathrm{SrCuO}_{2}$ on varying the lattice constant in the $c$ direction, keeping the in-plane lattice constants $a$ and $b$ fixed at $a_{\mathrm{STO}}$. When the structure is rotated so that $b$ and $c$ are forced to match $a_{\text {STO }}$ and $a$ is varied, the resulting strain energy is given by the black curve.

also be synthesized, ${ }^{30}$ only the infinite-layer structure is taken into consideration in thin-film growth experiments, ${ }^{21-23,31}$ thus we refer here to the infinite-layer structure as the bulk structure and neglect other possibilities in this Rapid Communication.

Including an on-site Coulomb repulsion term $U$ only slightly affects the calculated lattice constants, and can reproduce the observed antiferromagnetic insulating (AF-I) ground state ${ }^{32}$ with an energy gap located between filled oxygen $p$ bands and an unfilled minority-spin $\mathrm{Cu} d_{x^{2}-y^{2}}$ band. ${ }^{29}$ Because its nodes point toward neighboring oxygen atoms, the $d_{x^{2}-y^{2}}$ orbital hybridizes strongly with the oxygen $p$ states and is pushed up in energy. The resulting $\mathrm{Cu}^{2+} d^{9}$ configuration corresponds to all $\mathrm{Cu} d$ orbitals being filled except for the minority-spin $d_{x^{2}-y^{2}}$ orbital. This is apparent in the surfaces of constant magnetization density plotted in the left-hand panel of Fig. 1, where the spin density on the oxygen atoms also illustrates the AF superexchange coupling between neighboring $\mathrm{Cu}$ ions. The hybridization is mainly confined to the $\mathrm{CuO}_{2}$ plane and does not affect the ionic character of the $A^{2+}$ and $\mathrm{CuO}_{2}{ }^{-2}$ layers, so the electrostatic instability of $\mathrm{ACuO}_{2}$ thin films still exists by analogy with LAO thin films.

$\mathrm{ACuO}_{2}$ thin films. When SCO is grown on a STO substrate, it is assumed that positively charged $A^{2+}$ and negatively charged $\mathrm{CuO}_{2}{ }^{-2}$ layers alternate. ${ }^{21}$ Because this planar (" $\mathrm{CuO}_{2}$ ") structure will lead to an electrostatic instability, we also consider a chain-type ("CuO") structure that is formed by

TABLE I. Bulk lattice constants of perovskite $\mathrm{SrTiO}_{3}$ and infinitelayer copper oxides $A \mathrm{CuO}_{2}(A=\mathrm{Ca}, \mathrm{Sr}, \mathrm{Ba})$.

\begin{tabular}{lcccccc}
\hline \hline & $a^{\mathrm{LDA}}$ & $c^{\mathrm{LDA}}$ & $a^{\mathrm{GGA}}$ & $c^{\mathrm{GGA}}$ & $a^{\exp }$ & $c^{\exp }$ \\
\hline $\mathrm{SrTiO}_{3}$ & 3.87 & 3.87 & 3.95 & 3.95 & 3.905 & 3.905 \\
$\mathrm{CaCuO}_{2}$ & 3.77 & 3.08 & 3.87 & 3.20 & $3.853^{\mathrm{a}}$ & $3.177^{\mathrm{a}}$ \\
$\mathrm{SrCuO}_{2}$ & 3.84 & 3.38 & 3.95 & 3.47 & $3.926^{\mathrm{b}}$ & $3.432^{\mathrm{b}}$ \\
$\mathrm{BaCuO}_{2}$ & 3.92 & 3.68 & 4.03 & 3.84 & & \\
\hline
\end{tabular}

\footnotetext{
${ }^{\mathrm{a}}$ Reference 20.
}

${ }^{\mathrm{b}}$ Reference 19 .

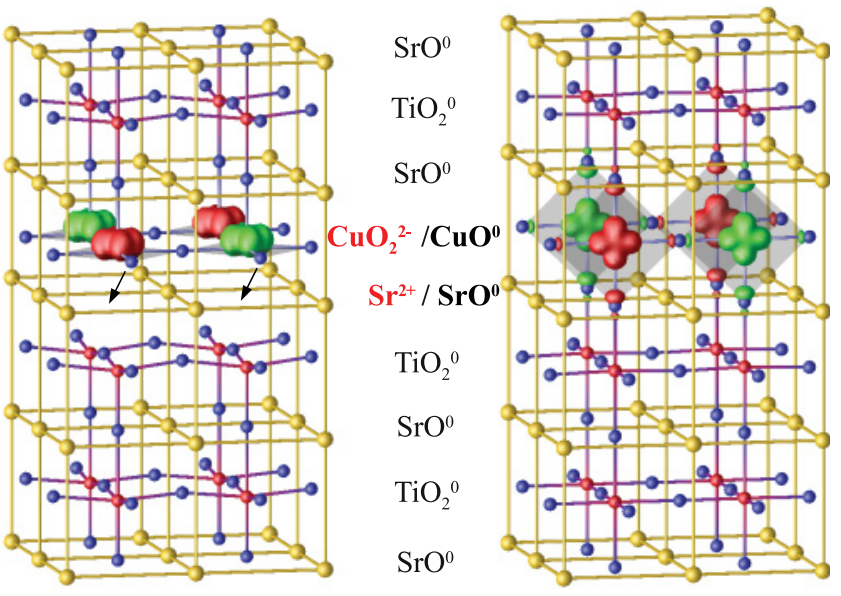

FIG. 2. (Color online) Relaxed structures and magnetic isosurfaces of $\mathrm{SrCuO}_{2} \mid \mathrm{SrTiO}_{3}$ multilayers with one unit cell of planar(left-hand panel) or chain-type (right-hand) $\mathrm{SrCuO}_{2}$ thin film. For simplicity, only three unit cells of $\mathrm{SrTiO}_{3}$ are shown. The arrows in the left-hand panel schematically indicate the oxygen displacements that transform the planar into a chain-type structure.

moving one oxygen atom from the $\mathrm{CuO}_{2}{ }^{2-}$ layer to the oxygen vacancy position in the $A^{2+}$ layers, as illustrated in Fig. 2 for the case of SCO|STO multilayers. This results in a thin film consisting of uncharged $\mathrm{SrO}^{0}$ and chain-type ${ }^{0}$ layers that does not suffer from an electrostatic instability but at the expense of strain (Fig. 1). For sufficiently thick films, we expect the planar structure to be most stable. It is not clear a priori what will occur for thin films of thickness $d$.

To answer this question, we calculate the energy difference $\Delta E=E^{\text {chain }}-E^{\text {planar }}$ as a function of $d$. As shown in Fig. 3, $\Delta E$ depends strongly on both $d$ and on the cation $A$. For $\mathrm{SrCuO}_{2}$ thin films grown on a lattice-matched STO substrate, it increases gradually from -1.2 to $+0.05 \mathrm{eV}$ per unit cell, as the thickness increases from one to six unit cells. Since both structures have the same stoichiometry, the sign of $\Delta E$

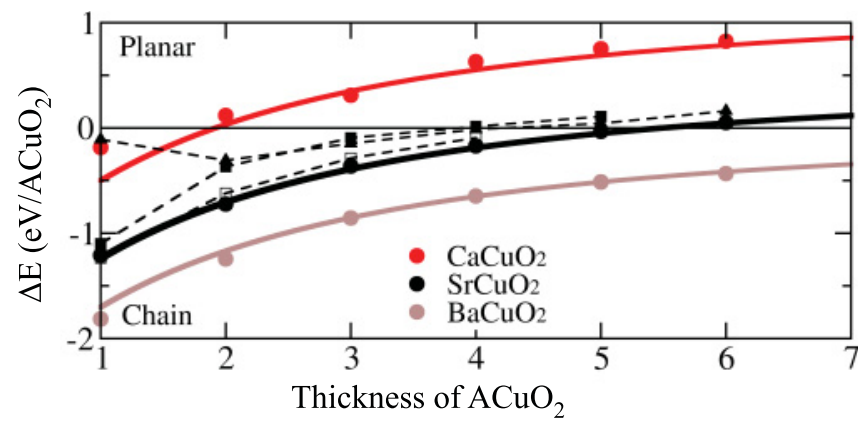

FIG. 3. (Color online) Total energy difference between planarand chain-type $A C O$ thin films as a function of the film thickness $d$. Symbols represent LDA results for $\mathrm{SrCuO}_{2}$ thin films in three forms: grown on a $\mathrm{SrTiO}_{3}$ substrate (black circles), as a multilayer with $\mathrm{SrTiO}_{3}$ (black squares), and freestanding (black triangles); the open squares represent LDA $+U$ results for multilayers. The solid black line for $\mathrm{SrCuO}_{2}$ is based on the analytical model including the electrostatic energy and strain energy discussed in the text, while the dark gray (red) and gray (brown) circles and solid lines differ only in terms of the calculated strain energies of $\mathrm{CaCuO}_{2}$ and $\mathrm{BaCuO}_{2}$. 
is a direct measure of their relative stability. Below a critical thickness of about five unit cells, chain-type thin films are energetically favorable; above it, planar-type films are more stable. In the cation series $\mathrm{Ca} \rightarrow \mathrm{Sr} \rightarrow \mathrm{Ba}, \Delta E(d)$ decreases but depends on thickness in the same way for all $A \mathrm{CuO}_{2}$ thin films. Similar behavior is found for multilayers and freestanding thin films, so surface or interface effects are not essential for a qualitative understanding. We then decompose $\Delta E(d)$ as $E_{C}-E_{P}$, where $E_{P}\left(E_{p}\right)$ denotes the electrostatic energy (density) induced by the instability of planar type thin films and $E_{C}$ is the chemical bonding energy caused by the oxygen atom moving to form a strained chain-type structure.

The planar thin films are terminated with positively charged $\mathrm{Sr}^{2+}$ and negatively charged $\mathrm{CuO}_{2}{ }^{2-}$ surfaces with nominal areal charge densities $\pm \sigma= \pm e / a^{2}$, where $a$ is the inplane lattice constant. They can be modeled as parallel-plate capacitors $5,6,12,13,17,23,33$ in which the plates are separated by a thickness $d$ of bulk material with dielectric constant $\varepsilon$. The electrostatic energy density associated with the electric field $\sigma / \varepsilon \varepsilon_{0}$ between the plates is $E_{p}^{0}=\sigma^{2} /\left(2 \varepsilon \varepsilon_{0}\right)$ or approximately $20 / \varepsilon \mathrm{eV}$ per unit cell, where $\varepsilon_{0}$ is the dielectric constant of vacuum. This constitutes a strong instability that will drive charge transfer that depends on the thickness and electronic structure of the thin films. ${ }^{23}$ If we assume a rigid flat-band approximation with constant density of states $\alpha$, the transferred charge is $\sigma /\left[1+a^{2} \varepsilon \varepsilon_{0} /(\alpha d)\right]$. As a result of the screening by the transferred charge, the residual electrostatic energy density becomes $E_{p}(d)=E_{p}^{0} /\left[1+\alpha d /\left(a^{2} \varepsilon \varepsilon_{0}\right)\right]^{2}$, where the screening factor clearly depends on $\alpha$ and thin-film thickness $d$. For a large gap insulator with $\alpha=0, E_{p}$ is a constant. ${ }^{34}$ For a good metal with a large $\alpha, E_{p}$ approaches zero. For a small-band-gap semiconductor with a moderate $\alpha, E_{p}$ vanishes when $d$ is large, consistent with bulk limits; if $d$ is reduced, less charge will be transferred and consequently $E_{p}$ will be enhanced. When the films are very thin, charge transfer cannot take place to quench the instability.

In that case, $E_{P}$ can be quenched by displacing oxygen atoms to form a chain-type structure as indicated in Fig. 2. Because the resulting structure corresponds to a strained $A \mathrm{CuO}_{2}$ thin film grown in a (100) orientation on a (001) STO substrate (right-hand side of Fig. 2), rather than with a conventional (001) orientation (left-hand side of Fig. 2), doing so will change the bonding energy $E_{C}$. The two types of thin film only differ in terms of strain. To estimate the strain energy of planar-type thin films, we fix the in-plane lattice constant of bulk $A \mathrm{CuO}_{2}$ at $a_{\mathrm{STO}}$ and determine the total energy as a function of $c$. For chain-type thin films, we consider $b$ and $c$ as the in-plane lattice constants, fix them to be equal to $a_{\mathrm{STO}}$, and minimize with respect to $a$, as shown in Fig. 1 . The energy difference per unit cell between the two minima is $E_{C}=0.45 \mathrm{eV}$ for $\mathrm{SrCuO}_{2}, 1.19 \mathrm{eV}$ for $\mathrm{CaCuO}_{2}$, and $-0.01 \mathrm{eV}$ for $\mathrm{BaCuO}_{2}$.

As shown in Fig. 3, $\Delta E(d)$ can be fit very well using these values for $E_{C}$ and values of $\alpha=0.1 e / \mathrm{V}$ and $\varepsilon=8$ in the modified parallel-plate capacitor model for $E_{P}$. The very good fit indicates that the competition between the two structures can be represented in terms of electronic and atomic reconstructions and that charge transfer can occur to partly screen the electrostatic instability without altering the atomic structures. However, as the films become thinner, the effect

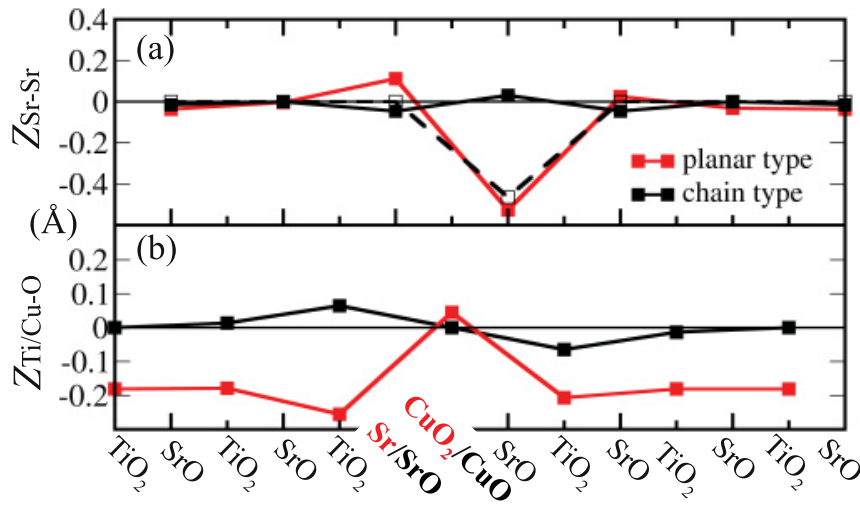

Layer index

FIG. 4. (Color online) (a) Spacings between Sr layers for SCO|STO multilayers with planar- and chain-type monolayers of SCO relative to $a_{\text {STO }}$ for bulk STO. The black open squares and dashed line represent ideal stacking without epitaxial strain. (b) The $\mathrm{Ti}-\mathrm{O}$ or $\mathrm{Cu}-\mathrm{O}$ corrugation in the $z$ direction for each layer in both types of SCO|STO multilayers.

of screening decreases. For fewer than about five unit cells of SCO on STO, the widely assumed planar-type thin film cannot be stabilized because of the instability. In spite of a considerable cost in strain energy, the chain-type thin film should become energetically favorable because of the lowering of the electrostatic polarization energy $E_{P}$.

Atomic and electronic structure. To discuss the atomic and electronic properties of the novel chain-type thin films, we focus on the multilayers shown in Fig. 2 with a single unit cell of SCO sandwiched between five unit cells of STO. The parallel behavior of the multilayer and thin-film curves (black circles and squares) in Fig. 3 indicates that the essential physics is the same, so we can dispense with discussing the effect of dangling bonds at the free surface. In the chain-type film, there are stiff $\mathrm{Cu}-\mathrm{O}$ bonds in the growth direction. Because of this, the multilayer containing chain-type SCO is almost $0.5 \AA$ longer in the $z$ direction than that containing planar SCO. This is illustrated in Fig. 4(a), where the spacing between layers containing $\mathrm{Sr}$ atoms is plotted. Measurement of this spacing should be possible and would provide experimental evidence for chain-type thin films. The presence of zigzag Ti-O buckling in Figs. 2 and 4(b) in the planar-type films is a strong indicator of internal electric fields ${ }^{11-13,17}$ that should be observable in structural studies. ${ }^{35}$

The ground state of the planar-type SCO film is an AF metal. Consistent with a previous LDA $+U$ calculation for $\mathrm{YBa}_{2} \mathrm{Cu}_{3} \mathrm{O}_{6}$ interfaces with STO, ${ }^{39}$ we find that the $d_{x^{2}-y^{2}}$ orbital character of the $\mathrm{Cu}^{2+} d^{9}$ minority-spin hole is largely unchanged by the polar nature of thin films and the presence of apical oxygen atoms in the STO substrate that leads to $\mathrm{Cu}-\mathrm{O}-\mathrm{Ti}$ type bonding. In contrast, the ground state of the chain-type film is an AF insulator with a localized magnetic moment of $0.6 \mu_{B}$ on $\mathrm{Cu}$ ions and an energy gap of $1.6 \mathrm{eV}$. The energy gap between filled $\mathrm{O} p$ states and an unfilled minority-spin $\mathrm{Cu}$ $d$ state will decrease on increasing the thickness of the SCO film. The unfilled $\mathrm{Cu} d$ orbital character will be $d_{z^{2}-y^{2}}$, as shown in Fig. 2, because the $\mathrm{Cu}$ ions are still in $\mathrm{a} \mathrm{CuO}_{4}$ square planar configuration surrounded by four oxygen atoms in the 
$y-z$ plane rather than the original, bulk $x-y$ plane. Our results suggest an explanation for the $d_{3 z^{2}-r^{2}}$ orbital observed at the $\mathrm{YBa}_{2} \mathrm{Cu}_{3} \mathrm{O}_{7} \mid \mathrm{LaMnO}_{3}$ interface ${ }^{7}{ }^{7}$ the electrostatic instability of $\mathrm{CuO}_{2}$ planes ${ }^{23,31}$ may be driving oxygen atoms (or vacancies) to move and effectively tilt the $\mathrm{CuO}_{4}$ square toward the $z$ direction, favoring orbitals pointing in that direction. The polar nature of $\mathrm{CuO}_{2}$ planes may play a key role in the structure and properties of ultrathin films and interfaces of HTS cuprates.

Conclusion. Using first-principles calculations, we predict that a polar electrostatic instability will lead to chain-type structures being preferred when films of $A \mathrm{CuO}_{2}$ are sufficiently thin, and interpret this with a parallel-plate capacitor model including charge transfer. As the polar film is made thinner, less charge can be transferred, the residual electrostatic energy increases, and formation of nonpolar chain-type structures becomes an energetically favorable way to resolve the instability without changing the stoichiometry. Similar considerations can be applied to LAO|STO. Although pure charge transfer can avoid the "polar catastrophe," 5,11 it cannot fully quench the electrostatic instability. A large residual electrostatic energy, especially in the low thickness LAO regime, will drive the formation of oxygen vacancies or other defects.

This work was supported by "NanoNed," a nanotechnology program of the Dutch Ministry of Economic Affairs. This work is also part of the research programs of "Stichting voor Fundamenteel Onderzoek der Materie" (FOM) and the use of supercomputer facilities was sponsored by the "Stichting Nationale Computer Faciliteiten" (NCF), both financially supported by the "Nederlandse Organisatie voor Wetenschappelijk Onderzoek" (NWO).
*Current address: Institute for Solid State Physics, Vienna University of Technology, Austria.

${ }^{1}$ S. Okamoto and A. J. Millis, Nature (London) 428, 630 (2004).

${ }^{2}$ A. Ohtomo and H. Y. Hwang, Nature (London) 427, 423 (2004).

${ }^{3}$ S. Thiel, G. Hammerl, A. Schmehl, C. W. Schneider, and J. Mannhart, Science 313, 1942 (2006).

${ }^{4}$ M. Huijben, A. Brinkman, G. Koster et al., Adv. Mater. 21, 1665 (2009).

${ }^{5}$ N. Nakagawa, H. Y. Hwang, and D. A. Muller, Nat. Mater. 5, 204 (2006).

${ }^{6}$ J. Goniakowski, F. Finocchi, and C. Noguera, Rep. Prog. Phys. 71, 016501 (2008).

${ }^{7}$ J. Chakhalian, J. W. Freeland, H.-U. Habermeier et al., Science 318, 1115 (2007)

${ }^{8}$ J. Junquera and P. Ghosez, Nature (London) 422, 506 (2003).

${ }^{9}$ Z. Zhong and P. J. Kelly, Europhys. Lett. 84, 27001 (2008).

${ }^{10}$ Z. S. Popović, S. Satpathy, and R. M. Martin, Phys. Rev. Lett. 101, 256801 (2008).

${ }^{11}$ R. Pentcheva and W. E. Pickett, Phys. Rev. Lett. 102, 107602 (2009).

${ }^{12}$ N. C. Bristowe, E. Artacho, and P. B. Littlewood, Phys. Rev. B 80, 045425 (2009).

${ }^{13}$ H. Chen, A. Kolpak, and S. Ismail-Beigi, Phys. Rev. B 82, 085430 (2010).

${ }^{14}$ G. Herranz, M. Basletić, M. Bibes et al., Phys. Rev. Lett. 98, 216803 (2007).

${ }^{15}$ A. S. Kalabukhov, Y. A. Boikov, I. T. Serenkov et al., Phys. Rev. Lett. 103, 146101 (2009).

${ }^{16}$ S. A. Chambers, M. H. Engelhard, V. Shutthanandan et al., Surf. Sci. Rep. 65, 317 (2010).

${ }^{17}$ Z. Zhong, P. X. Xu, and P. J. Kelly, Phys. Rev. B 82, 165127 (2010).

${ }^{18}$ T. Siegrist, S. M. Zahurak, D. W. Murphy, and R. S. Roth, Nature (London) 334, 231 (1988).

${ }^{19}$ M. Takano, Y. Takeda, H. Okada, M. Miyamoto, and T. Kusaka, Physica C 159, 375 (1989).

${ }^{20}$ N. Kobayashi, Z. Hiroi, and M. Takano, J. Solid State Chem. 132, 274 (1997).
${ }^{21}$ J.-M. Triscone and O. Fischer, Rep. Prog. Phys. 60, 1673 (1997).

${ }^{22}$ M. Huijben et al., e-print arXiv:cond-mat/1008.1896 (unpublished).

${ }^{23}$ A. J. Millis and D. G. Schlom, Phys. Rev. B 82, 073101 (2010).

${ }^{24}$ E. Bousquet, M. Dawber, N. Stucki et al., Nature (London) 452, 732 (2008).

${ }^{25}$ N. A. Benedek and C. J. Fennie, Phys. Rev. Lett. 106, 107204 (2011).

${ }^{26}$ P. E. Blöchl, Phys. Rev. B 50, 17953 (1994).

${ }^{27}$ G. Kresse and D. Joubert, Phys. Rev. B 59, 1758 (1999).

${ }^{28}$ S. L. Dudarev, G. A. Botton, S. Y. Savrasov, C. J. Humphreys, and A. P. Sutton, Phys. Rev. B 57, 1505 (1998).

${ }^{29}$ V. I. Anisimov, J. Zaanen, and O. K. Andersen, Phys. Rev. B 44, 943 (1991).

${ }^{30}$ N. Motoyama, H. Eisaki, and S. Uchida, Phys. Rev. Lett. 76, 3212 (1996).

${ }^{31}$ G. Koster, A. Brinkman, H. Hilgenkamp et al., J. Phys. Condens. Matter 20, 264007 (2009).

${ }^{32}$ D. Vaknin, E. Caignol, P. K. Davies, J. E. Fischer, D. C. Johnston, and D. P. Goshorn, Phys. Rev. B 39, 9122 (1989).

${ }^{33}$ J. Goniakowski and C. Noguera, and L. Giordano, Phys. Rev. Lett. 98, 205701 (2007).

${ }^{34}$ Consistent with "uncompensated polarity" in $\mathrm{MgO}$ films—see Ref. 33.

${ }^{35}$ Theoretical studies for $\mathrm{MgO}(111)$ (Ref. 36) and $\mathrm{ZnO}$ (Ref. 37) polar thin films predict analogous changes in atomic structures driven by electrostatic instabilities. Such changes have recently been observed (Ref. 38).

${ }^{36}$ J. Goniakowski, C. Noguera, and L. Giordano, Phys. Rev. Lett. 93, 215702 (2004).

${ }^{37}$ C. L. Freeman, F. Claeyssens, N. L. Allan, and J. H. Harding, Phys. Rev. Lett. 96, 066102 (2006).

${ }^{38}$ C. Tusche, H. L. Meyerheim, and J. Kirschner, Phys. Rev. Lett. 99, 026102 (2007).

${ }^{39}$ N. Pavlenko, I. Elfimov, T. Kopp, and G. A. Sawatzky, Phys. Rev. B 75, 140512 (2007). 